\title{
Congenital bronchopulmonary malformation: CT histopathological correlation
}

\author{
Martin Kyncla , Martin Kocia, Lea Ptackovaa ${ }^{a}$ Ludmila Hornofova ${ }^{b}$, Ondrej Fabian ${ }^{b}$, Jiri Snajdaufc, Miloslav Rocek, \\ Marcela Pychovac
}

Background. This study evaluated the accuracy of postnatal computed tomography (CT) imaging in the identification of congenital bronchopulmonary malformation (BPM) in comparison with histopathological analysis.

Methods. CT scans of prenatally diagnosed BPMs from 24 patients with available histology were analysed retrospectively. The CT images were reviewed blinded to histological findings by two radiologists. Specific diagnosis was assigned based on predetermined criteria. The accuracy of CT was evaluated.

Results. The agreement rate in CT diagnosis between two radiologists was 100\%. In $75 \%$ the lesions were located in the lower lobes. An overlap of $71 \%$ in CT and histopathological diagnoses was reached. The least matching diagnosis was type 2 CPAM.

Conclusion. Contrast enhanced chest $\mathrm{CT}$ is very accurate in characterizing the BPM spectrum and provides important information on lesion type and structure.

Key words: bronchopulmonary malformation, computed tomography, histopathological findings, predetermined criteria

Received: June 5, 2016; Accepted with revision: September 27, 2016; Available online: October 17, 2016 http://dx.doi.org/10.5507/bp.2016.051

${ }^{a}$ Department of Radiology, $2^{\text {nd }}$ Faculty of Medicine, Charles University in Prague and Faculty Hospital Motol, Prague, Czech Republic ${ }^{b}$ Department of Pathology and Molecular Medicine, $2^{\text {nd }}$ Faculty of Medicine, Charles University in Prague and Faculty Hospital Motol, Prague, Czech Republic

'Department of Paediatric Surgery, $2^{\text {nd }}$ Faculty of Medicine, Charles University in Prague and Faculty Hospital Motol, Prague, Czech Republic Corresponding author: Martin Kyncl, e-mail:martin.kyncl@fnmotol.cz

\section{INTRODUCTION}

Bronchopulmonary malformations refer to a spectrum of pulmonary developmental anomalies, including congenital pulmonary airway malformation (CPAM), previously known as congenital cystic adenomatoid malformation (CCAM), bronchopulmonary sequestration (BPS), hybrid lesion and congenital lung overinflation (CLO). These anomalies consist of about $90 \%$ of those seen in clinical practise ${ }^{1}$. The advent of prenatal US and MRI has considerably increased the prenatal diagnosis of lung pathologies but complete characterization remains difficult. In utero airway maldevelopment with resultant obstruction is a recurrent proposed aetiology for many of these lesions, especially those associated with hyperlucent or cystic lung changes ${ }^{2}$. This causes even more confusion in terms of how these malformations should be described ${ }^{3}$. Because terminology remains a problem, the use of a descriptive approach is strongly supported in practice ${ }^{4,5}$. In patients with clinical suspicion of BPM, a chest $\mathrm{CT}$ is required for the confirmation of diagnosis and further characterization of the lesion ${ }^{6}$. The aim of our study was to assess the accuracy of CT in classifying different types of BPMs and to correlate the imaging with pathological findings. We also provide a description of a CT paediatric protocol and technical factors that may optimize the evaluation of these lesions.

\section{MATERIALS AND METHODS}

We identified 24 patients referred to our institution between the years 2010 and 2015 with a prenatal ultrasound diagnosis of BPM who had undergone surgical resection. Postnatal chest CT scans of these patients performed prior to surgery was the basis of this retrospective study. CT studies were obtained on an Aquillion 64 CT system (Toshiba, Japan); CT scans were carried out without a contrast agent in 7 cases and with intravenous administration of an iodinated contrast agent in 17 cases. Contrast chest CTs were performed according to a dedicated paediatric protocol using $80 \mathrm{kV}$ and $40 \mathrm{~mA}$ with automated tube current modulation, collimation of $1.5 \mathrm{~mm}$, a corrected helical pitch of 0.828 , a gantry rotation time of $0.5 \mathrm{~s}$, and a mechanical power injector for contrast administration. We used a non-ionic low-osmolar contrast medium at a volume of $2 \mathrm{~mL} / \mathrm{kg}$ of body weight and a flow rate of 0.8-1.5 mL/s. We used visual bolus tracking with the region of interest in the upper abdominal aorta. Anatomic scan coverage was positioned from the level of the thoracic inlet to the diaphragm. Neonates and young infants were scanned under sedation. Post-processing used mediastinal and lung windows settings; multiplanar volume reformation (MPR) with a slice thickness of up to $0.5 \mathrm{~mm}$. Two radiology residents with 2 years of experience reviewed $24 \mathrm{CT}$ scans. The criteria for re-evaluation were set by a senior radiology consultant with 20 years of 


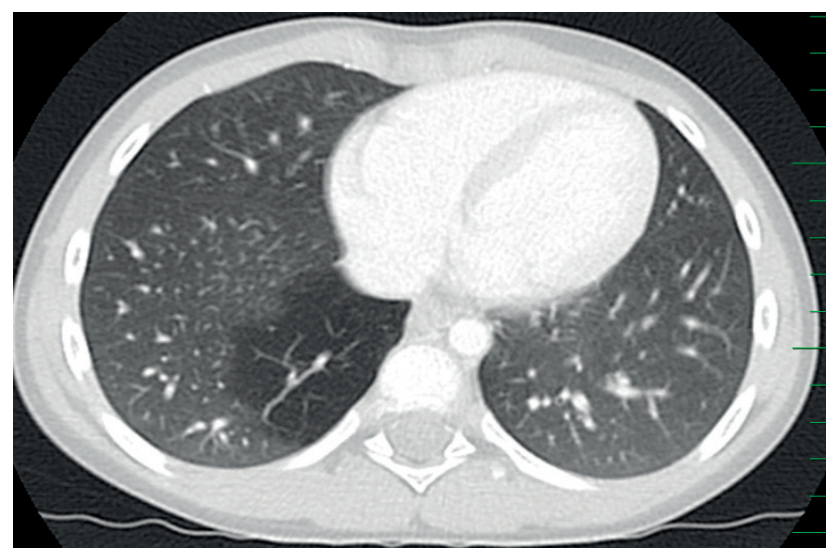

Fig. 1. Focal area of pulmonary hypoattenuation in the right lower lobe (LLL), congenital lung overinflation (CLO).

experience in paediatric imaging who mentored the residents. The radiology residents blinded to the histopathological results reviewed the CT images independently and recorded the site of the lesion, the presence and size of cysts, the presence or absence of a feeding systemic vessel, areas of overinflation, and solid lesions. Postnatal CT lung lesion diagnosis was compared with the diagnosis provided by histopathology. The CT detected lesions were classified by predetermined criteria. A lung lesion was considered CPAM type 1 (Stocker's ${ }^{7}$ classification) when the cystic component of the lesion was greater than $2 \mathrm{~cm}$, CPAM type 2 if the cyst $\leq 2 \mathrm{~cm}$, and CPAM type 3 if a solid lesion was present. Lung lesions with a feeding vessel were classified as BPS. Lesions with CPAM (1-3) characteristics with a feeding vessel were diagnosed as hybrid lesions. Lung lesions with lobar, segmental or subsegmental hypoattenuation were considered as CLO when these characteristics were present separately. This was referred to as an additional characteristic of other lesions when combined with CPAMs or BPS. These data were correlated with surgical findings (if available) and histopathological results.

\section{RESULTS}

There were 24 patients ( 16 females and 8 males) with a median age of 4.3 years at surgical resection (an interquartile range of 1.1-5.4 years). The median interval between $\mathrm{CT}$ study and surgical resection was 55 days (an interquartile range of 14-128 days). According to the defined criteria, there was complete agreement between two radiologists in determining the final CT diagnosis. Overall, the radiologists identified six congenital lung overinflations, six were type 2 CPAMs, five were type 1 CPAMs, five were bronchopulmonary sequestrations, one was type 3 CPAM, and one presented criteria that were not fully applicable.

The CT results are summarized in Table 1. As shown, out of 24 lesions, 18 (75\%) were located in the lower lobes, just one (4\%) in the right middle lobe and $5(21 \%)$ in upper lobes. In $75 \%$ cases ( 18 out of 24 ) cases the areas of

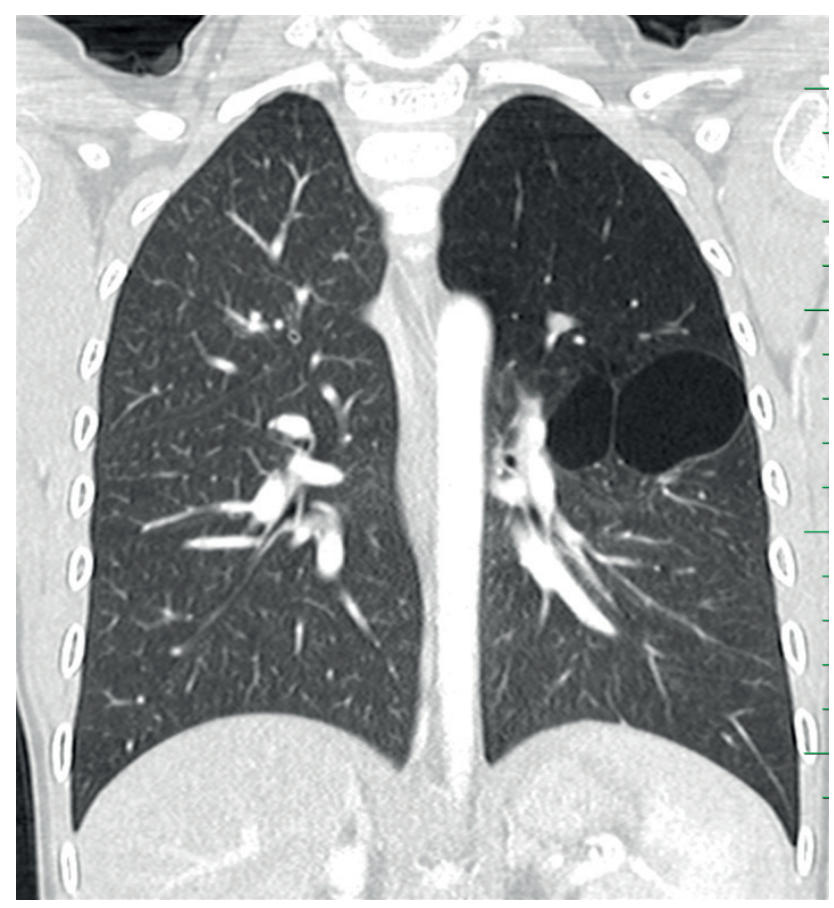

Fig. 2. Multiple large cysts measured $>2 \mathrm{~cm}$ in the left upper lobe (LUL), with adjacent area of hypoattenuation, mixed lesion with CPAM 1 and CLO component. Coronal postcontrast CT reconstruction, lung window.

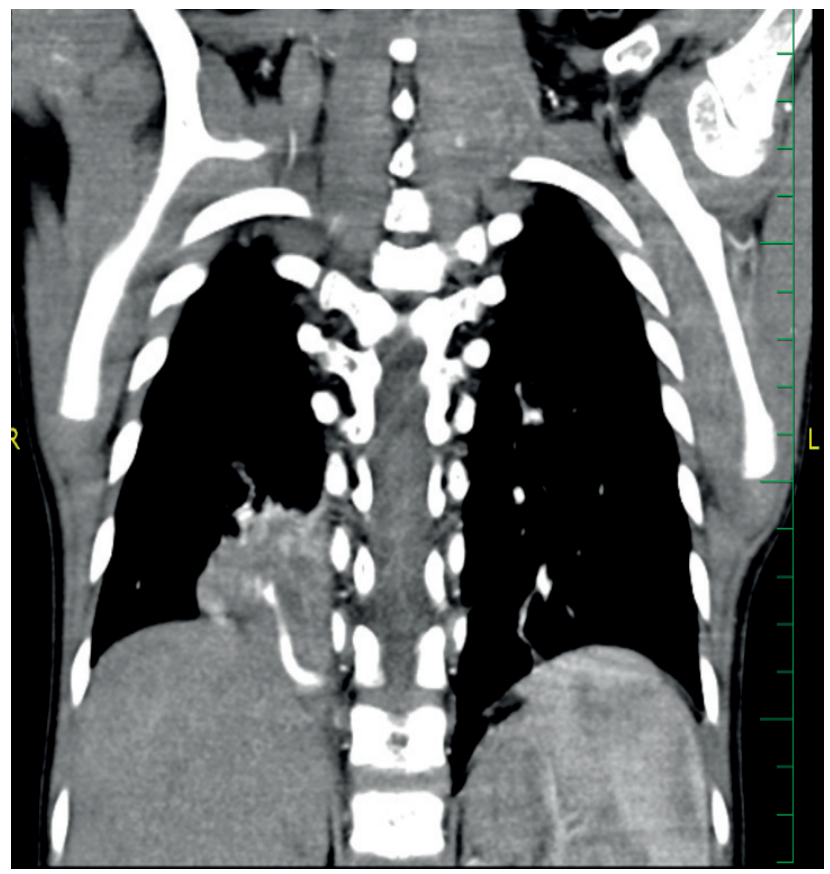

Fig. 3. A coronal CT reconstruction, mediastinal windowing, shows contrast enhanced a systemic arterial feeder to the pulmonary sequestration of the right lower lobe.

hypoattenuation were present (Fig. 1) which correspond with the findings reported ${ }^{2,5,8,9}$. In $50 \%$ cases (12 out of 24) the areas of hypoattenuation occurred together with another type of BPMs, with three bronchopulmonary sequestrations, all being type 1 CPAMs, and with four sequestrations of type 2 CPAMs, which is an additional CT characteristic of the lesion. Post-inflammatory changes 
were very common (not shown in the tables) and were recorded in ten cases.

The comparison of CT diagnosis and histopathological results is shown in Table 2. A diagnosis overlap of 71\% (17 cases out of 24) between CT and histology was observed. Histopathology confirmed five out of six CLO diagnoses, whereas one case was histologically classified as type 2 CPAM. Only three out of six type 2 CPAMs diagnosed at CT were confirmed by histology, for the other three the conclusion was different; in the first case of mismatch, the pathologists were unable to distinguish type 1 and type 2 CPAM, in the second case they concluded type 1 CPAM, and in the third case they changed the diagnosis to BPS given the finding of large atypical vessels in the sample. Type 1 CPAMs (Fig. 2) were confirmed four times; in one case the histological diagnosis was changed to type 2 CPAM. All cases of BPS (Fig. 3) were confirmed by histology. Only one case of type 3 CPAM (case No. 1) was diagnosed with a CT scan. However, this lesion resembled BPS but no feeding vessel was clearly identified. Type 3 CPAM was not confirmed and after histological examination the diagnosis was changed to a hybrid lesion. In case No. 16 the predefined criteria were not fully applicable. A more than $3 \mathrm{~cm}$ cystic lesion with fluid content

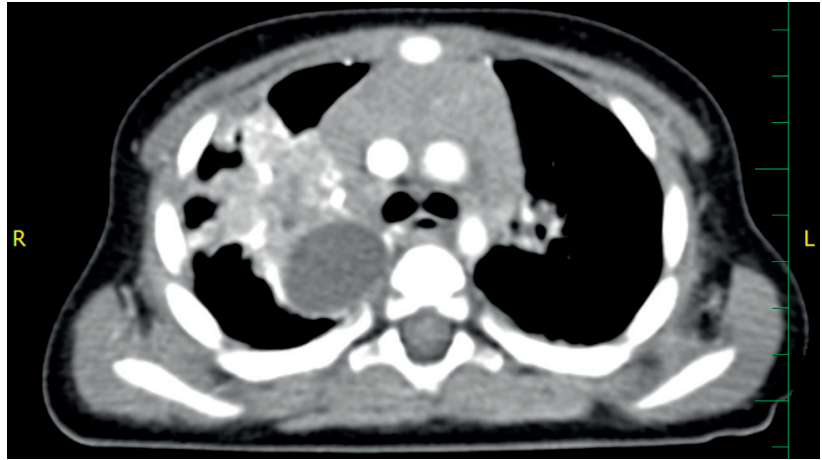

Fig. 4. The axial contrast CT scan demonstrates a foregut malformation, esophageal cyst, in the right hemithorax.

was observed in the medial wall of the right upper lung. The lesion was therefore classified as a bronchogenic cyst. However, histopathology established the final diagnosis of an oesophageal cyst with extensive purulent destructive changes to the tissues of the bronchial tree (Fig. 4). This particular lesion was misdiagnosed as CPAM at a referring prenatal ultrasound. According to the defined criteria, type 2 CPAM diagnosis was most frequently misdiagnosed as BPM during CT examination.

Table 1. Imaging characteristics of BPMs.

\begin{tabular}{|c|c|c|c|c|c|c|c|}
\hline Case & $\begin{array}{l}\text { Age at surgery } \\
\text { (years) }\end{array}$ & Sex & CT diagnosis & Site & Character & $\begin{array}{c}\text { Area } \\
\text { of hypoattenuation }\end{array}$ & $\begin{array}{c}\text { Feeding } \\
\text { vessel }\end{array}$ \\
\hline 1 & 6.1 & $\mathrm{M}$ & CPAM 3 & LLL & Solid w/o cysts & No & No \\
\hline 2 & 4.0 & $\mathrm{~F}$ & CPAM 1 & LLL & Cysts $>2 \mathrm{~cm}$ & Yes & No \\
\hline 3 & 0.7 & $\mathrm{~F}$ & CPAM 2 & RLL & Cysts $\leq 2 \mathrm{~cm}$ & No & No \\
\hline 4 & 1.2 & $\mathrm{~F}$ & CPAM 2 & RML & Cysts $\leq 2 \mathrm{~cm}$ & Yes & No \\
\hline 5 & 7.2 & M & CPAM 2 & RLL & Cysts $\leq 2 \mathrm{~cm}$ & Yes & No \\
\hline 6 & 1.3 & $\mathrm{~F}$ & CPAM 1 & RUL & Cysts $>2 \mathrm{~cm}$ & Yes & No \\
\hline 7 & 4.5 & $\mathrm{~F}$ & BPS & RLL & Solid w/o cysts & No & Yes \\
\hline 8 & 5.4 & M & CPAM 1 & LUL & Cysts $>2 \mathrm{~cm}$ & Yes & No \\
\hline 9 & 5.4 & $\mathrm{~F}$ & CPAM 2 & LLL & Cysts $\leq 2 \mathrm{~cm}$ & Yes & No \\
\hline 10 & 4.9 & M & CLO & RLL & No cysts & Yes & No \\
\hline 11 & 8.1 & $\mathrm{~F}$ & CPAM 1 & RLL & Cysts $>2 \mathrm{~cm}$ & Yes & No \\
\hline 12 & 3.7 & $\mathrm{~F}$ & BPS & RUL & Solid w/o cysts & Yes & Yes \\
\hline 13 & 0.1 & $\mathrm{~F}$ & BPS & RLL & Solid w/o cysts & No & Yes \\
\hline 14 & 5.1 & $\mathrm{~F}$ & CPAM 1 & LUL & Cysts $>2 \mathrm{~cm}$ & Yes & No \\
\hline 15 & 5.2 & $\mathrm{~F}$ & CPAM 2 & RLL & No cysts & Yes & No \\
\hline 16 & 0.6 & M & $\mathrm{BC}$ & RUL & Cysts $>2 \mathrm{~cm}$ & No & No \\
\hline 17 & 5.6 & $\mathrm{~F}$ & BPS & LLL & No cysts & Yes & Yes \\
\hline 18 & 4.9 & $\mathrm{~F}$ & CLO & RLL & No cysts & Yes & No \\
\hline 19 & 0.3 & M & CLO & LLL & No cysts & Yes & No \\
\hline 20 & 3.8 & M & BPS & LLL & No cysts & Yes & Yes \\
\hline 21 & 2.0 & $\mathrm{~F}$ & CLO & LLL & No cysts & Yes & No \\
\hline 22 & 0.8 & M & CLO & RLL & No cysts & Yes & No \\
\hline 23 & 0.1 & $\mathrm{~F}$ & CPAM 2 & LLL & Cysts $\leq 2 \mathrm{~cm}$ & No & No \\
\hline 24 & 5.5 & $\mathrm{~F}$ & CLO & RLL & No cysts & Yes & No \\
\hline
\end{tabular}

CPAM, congenital pulmonary airway malformation; BPS, bronchopulmonary sequestration; CLO, congenital lung onverinflation; BC, bronchial cyst; LLL, left lower lobe; LUL, left upper lobe; RLL, right lower lobe; RML, right middle lobe; RUL, right upper lobe;

a Lesion in patient 16 consisted of a solid part and one cyst $30 \mathrm{~mm}$ in diameter with fluid content; it was located in RUL in paratracheal/paraesophageal location and therefore was concluded as bronchial cyst with secondary postinfectious changes. Histopathology changed the final diagnosis to an esophageal cyst. 
Table 2. Comparison of CT diagnosis and histopathological results.

\begin{tabular}{|c|c|c|c|c|c|c|c|}
\hline \multirow{2}{*}{ Histopathology } & \multicolumn{7}{|c|}{ CT diagnosis } \\
\hline & Bronchial cyst & BPS & CLO & CPAM 1 & CPAM 2 & CPAM 3 & Total \\
\hline BPS & & 5 & & & 1 & & 6 \\
\hline CLO & & & 5 & & & & 5 \\
\hline CPAM 1 & & & & 4 & 1 & & 5 \\
\hline CРAM $1 / 2$ & & & & & 1 & & 1 \\
\hline CPAM 2 & & & 1 & 1 & 3 & & 5 \\
\hline Esophageal cyst & 1 & & & & & & 1 \\
\hline Hybrid & & & & & & 1 & 1 \\
\hline Total & 1 & 5 & 6 & 5 & 6 & 1 & 24 \\
\hline
\end{tabular}

\section{DISCUSSION}

The BPM complex consists of a broad spectrum of developmental abnormalities, ranging from abnormal lungs with normal vasculature to abnormal vasculature with normal lungs and lesions with both parenchymal and vascular abnormalities ${ }^{10}$. The pathogenesis of these lesions is in continuous evolution. Langston ${ }^{5}$ suggested that many of the bronchopulmonary anomalies represent an obstruction malformation sequence with secondary pulmonary dysplastic changes. Differences in the level of airway obstruction, the completeness of the obstruction, and the timing of obstructive events may act together to produce varied patterns of malformations. One theory is that that these lesions are related to airway obstruction with secondary pulmonary dysplastic changes. Kunisaki ${ }^{8}$ and others ${ }^{5}$ claimed that many congenital bronchopulmonary malformations may represent a continuum of foetal lung anomalies associated with unrecognized airway obstruction. Therefore, for example, bronchial atresia occurring early in gestation might favour CPAM or a bronchogenic cyst, while obstruction developing later in gestation might predispose the lungs to develop BPS or CLO (ref. ${ }^{5}$ ). Some authors ${ }^{2}$ suggest that these anomalies have been characterized as lesions of defective budding, differentiation and separation of primitive foregut. The abnormal connection of the tubular components of the lung forms the basis of all congenital malformations. The entities included are CPAM, pulmonary sequestration, bronchogenic cyst, bronchial atresia, and CLO. Other anomalies, such as pulmonary agenesis, aplasia, hypoplasia, tracheal bronchus, oesophageal and neurenteric cysts, oesophageal and tracheal atresia, tracheoesophageal fistula, and other connections between the gastrointestinal tract and the lung can also be considered part of bronchopulmonary foregut malformations. A genetic cause of BPM has also been proposed and some authors ${ }^{11}$ have hypothesized that CPAMs and pleuropulmonary blastoma (PPB), despite their clinical and histopathological heterogeneity, are caused by genetic defects which might disrupt common molecular pathways controlling airway formation. Many neonates with prenatally diagnosed BPM, regardless of the underlying pathology, will be asymptomatic. BPMs that apparently completely involute in utero are documented to be still present when CT-examined after birth. Many of these also have a normal appearance on radiological examination $^{12,13}$. A number of lesions will remain asymptomatic but there remains a small risk of neoplasia associated with some forms of cystic lung disease. Many of these lesions exhibit more than one component, possibly due to a common origin. Since bronchial obstruction occurring during early lung development may be an inciting event in the disease pathogenesis of CPAMs (ref. ${ }^{5-8}$ ). Some authors have postulated that an obstructing bronchial mucus plug may transiently induce abnormal pulmonary growth, thereby mimicking a lung malformation until the obstruction is alleviated ${ }^{14}$. It is also conceivable that these lesions may outgrow their existing blood supply or, as in the case of extralobar BPS, undergo spontaneous torsion towards the end of pregnancy ${ }^{15}$. CLO is lung segment overinflation characterized during microscopic analysis by air space enlargement without maldevelopment ${ }^{5}$. Overinflation accounts for approximately $20 \%$ of all prenatally diagnosed lethal lung malformations ${ }^{1}$. Pathologically, CLO is divided into two subgroups; the first (previously known as congenital lobar emphysema or CLE) is associated with an overinflated lung lobe caused by its extrinsic or intrinsic abnormality or compression, the second group is characterized by lobar, segmental, or subsegmental overinflation and high association with bronchial atresia ${ }^{16}$. CLO occurring initially after delivery may appear like an area of increased opacity related to retained foetal fluid. It is preferable not to image infants during the immediate postdelivery period ${ }^{1}$. There is now even more confusion as to how these malformations should be described ${ }^{3}$. Therefore we, like some other autors ${ }^{1-3}$, prefer to describe radiological findings thoroughly rather than try to categorize lesions in terms of pathologic terminology only. From a radiologist's point of view it is essential to realize what is actually seen on a CT image and what should be recorded without the undertone of embryological speculation. Radiological and histopathological descriptions should be kept separate ${ }^{3}$. Important findings include localization of an involved pulmonary lobe, depiction of vascular supply and the presence or absence of aberrant arterial feeder, description of the internal components of the lesion, solid and cystic portion, and the degree and character of aeration. According to the defined criteria, there was a complete agreement between two radiologists in the final CT diagnosis. It is advisable to evaluate 
BPMs according to these relatively easy-to-follow criteria. Our patient series allowed for the imaging modality of postnatal CT to be used to monitor the BPM complex to be then compared with histopathological data. In our series and according to histopathological findings, the CT was accurate in all cases of BPS. Lanza et al. (ref. ${ }^{17}$ ) found concordance between $\mathrm{CT}$ and histopathology in $66.7 \%$, but their series was small and focused only on CPAMs. Zeidan et al. (ref. ${ }^{18}$ ) affirmed the accuracy of $\mathrm{CT}$ in all cases of CPAM, but only in 5 out of 6 patients in BPS. In our study, there was concordance in the detection of histologically confirmed CPAMs. Disagreements between CT and histopathological findings were caused primarily by to the inclusion of the CPAM types 1, 2 and 3 in our predetermined criteria. Type 2 CPAMs are the most frequent cause of mismatched diagnosis. We also observed a higher incidence of mixed lesions with coincidence of BPMs and the areas of hypoattenuation on CT findings. For the purpose of the study, these changes were called the areas of hypoattenuation, but we believe they represent an overlapping spectrum with CLO and other BPMs. Histopathologists might not notice these changes in a histologic specimen. This finding may be in accordance with the proposed pathogenesis of congenital lung lesions and the overlapping spectrum of an airway obstruction malformation sequence. Dedicated pathologists should evaluate the specimens of BPMs as diagnoses are often confusing and speculative when made without appropriate experience and knowledge. In our opinion, consensus and mutual cooperation between radiologists and histopathologists are essential in determining the final diagnosis of BPMs. Imaging methods are crucial for obtaining correct histopathological results. Contrast CT was accurate in all cases of the visualization of the aberrant systemic vessel. Additional studies including larger cohorts of patients are necessary to validate our findings.

\section{CONCLUSION}

Postnatal contrast chest CT is considered the most accurate examination in the postnatal radiological investigation of pulmonary lesions diagnosed prenatally. Hyperlucent lesions and CLOs presuppose an overlapping spectrum of an airway obstruction malformation sequence. Consensus and cooperation between radiologists and histopathologists are of paramount importance.

Acknowledgement: Supported by the project (Ministry of Health, Czech Republic) for the conceptual development of research organizations 00064203 (University Hospital in Motol, Prague, Czech Republic)

Author contribution: All authors contributed equally to preparing the manuscript.

Conflict of interest statement: The authors state that there are no conflicts of interest regarding the publication of this article.

\section{REFERENCES}

1. Epelman M, Kreiger P, Servaes S, Victoria T, Hellinger J. Current imaging of prenatally diagnosed congenital lung lesions. Semin Ultrasound, CT MRI 2010;31(2):141-57. doi:10.1053/j.sult.2010.01.002

2 Newman B. Congenital bronchopulmonary foregut malformations: concepts and controversies. Pediatr Radiol 2006;36(8):773-91. doi:10.1007/s00247-006-0115-4

3. Bush A. Congenital lung disease: A plea for clear thinking and clear nomenclature. Pediatr Pulmonol 2001;32(4):328-37. doi:10.1002/ ppul.1126

4. Fowler D, Gould S. The pathology of congenital lung lesions. Semin Pediatr Surg 2015;24(4):176-82. doi:10.1053/j.sempedsurg.2015.02.002

5. Langston C. New Concepts in the Pathology of Congenital Lung Malformations. 2003;12(1):17-37.

6. Lee E, Boiselle P, Cleveland R. Multidetector CT evaluation of congenital lung anomalies. Radiology 2008;247(3):632-48. doi:10.1148/ radiol.2473062124

7. Stocker J, Madewell J, Drake R. Congenital cystic adenomatoid malformation of the lung. Classification and morphologic spectrum. Hum Pathol 1977;8(2):155-71. http://www.ncbi.nlm.nih.gov/ pubmed/856714. Accessed April 18, 2016.

8. Kunisaki S, Fauza D, Nemes L, Barnewolt CE, Estroff JA, Kozakewich HP, Jennings RW. Bronchial atresia: The hidden pathology within a spectrum of prenatally diagnosed lung masses. J Pediatr Surg 2006;41(1):61-5. doi:10.1016/j.jpedsurg.2005.10.082

9. Riedlinger W, Vargas $S$, Jennings R, Estroff JA, Barnewolt CE, Lillehei CW, Wilson JM, Colin AA, Reid LM, Kozakewich HP. Bronchial atresia is common to extralobar sequestration, intralobar sequestration, congenital cystic adenomatoid malformation, and lobar emphysema. Pediatr Dev Pathol 2006;9(5):361-73. doi:10.2350/06-01-0023.1

10. Pacharn P, Kline-Fath B, Calvo-Garcia M, Linam LE, Rubio El, Salisbury S, Brody AS. Congenital lung lesions: Prenatal MRI and postnatal findings. Pediatr Radiol 2013;43(9):1136-43. doi:10.1007/s00247013-2668-3

11. Boucherat O, Jeannotte L, Hadchouel A, Delacourt C, Benachi A. Title: Pathomechanisms of Congenital Cystic Lung Diseases: Focus on Congenital Cystic Adenomatoid Malformation and Pleuropulmonary Blastoma. 2016;16:62-8. doi:10.1016/j.prrv.2015.11.011

12. Koci M. Espr 2015. Pediatr Radiol 2015;45(S2):332. doi:10.1007/ s00247-015-3337-5

13. Bush A, Hogg J. Cystic lung lesions - prenatal diagnosis and management. Prenat Diagn 2008;28(10):604-11. doi:10.1002/pd.2039

14. Meizner I, Rosenak D. The vanishing foetal intrathoracic mass: consider an obstructing mucous plug. Ultrasound Obstet Gynecol 1995;5(4):275-7. doi:10.1046/j.1469-0705.1995.05040275.x

15. Kunisaki S, Ehrenberg-Buchner S, Dillman J, Smith E, Mychaliska G, Treadwell M. Vanishing foetal lung malformations: Prenatal sonographic characteristics and postnatal outcomes. J Pediatr Surg 2015;50(6):978-82. doi:10.1016/j.jpedsurg.2015.03.025

16. Barth R. Airway Anomalies, Lung and Mediastinal Masses, and Hydrothorax. In: Kline-Fath B, ed. Fetal Imaging Ultrasound and MRI 1st ed. Philadelphia, PA: Wolters Kluwer Health; 2015:596-601.

17. Lanza C, Bolli V, Galeazzi V, Fabrizzi B, Fabrizzi G. Malformazione adenomatoide-cistica in et pediatrica: Correlazione tra aspetti TC e reperti istopatologici. Radiol Medica 2007;112(4):612-9. doi:10.1007/ s11547-007-0166-0

18. Zeidan S, Gorincour G, Potier A, Ughetto F, Dubus JC, Chrestian MA, Grosse C, Gamerre M, Guys JM, de Lagausie P. Congenital lung malformation: evaluation of prenatal and postnatal radiological findings. Respirology 2009;14(7):1005-11. doi:10.1111/j.14401843.2009.01591.x 